\title{
Exercise improves quality of life in androgen deprivation therapy- treated prostate cancer: systematic review of randomised controlled trials
}

\author{
Laisa Teleni ${ }^{1,2}$, Raymond J Chan ${ }^{1,3}$, Alexandre Chan ${ }^{4,5}$, Elisabeth A Isenring ${ }^{2,6}$, \\ Ian Vela 7,8, Warrick J Inder ${ }^{9,10}$ and Alexandra L McCarthy ${ }^{1,11,12}$ \\ ${ }^{1}$ School of Nursing, Queensland University of Technology, Brisbane, Queensland, Australia \\ ${ }^{2}$ Faculty of Health Sciences and Medicine, Bond Institute of Health and Sport, Bond University, 2 Promethean Way, \\ Gold Coast, Queensland 4226, Australia \\ ${ }^{3}$ West Moreton Hospital and Health Service, Brisbane, Queensland, Australia \\ ${ }^{4}$ Department of Pharmacy, Faculty of Science, National University of Singapore, Singapore, Singapore \\ ${ }^{5}$ Oncology Pharmacy, National Cancer Centre Singapore, Singapore, Singapore \\ ${ }^{6}$ Department of Nutrition and Dietetics, Princess Alexandra Hospital, Brisbane, Queensland, Australia \\ ${ }^{7}$ Department of Urology, Princess Alexandra Hospital, Brisbane, Queensland, Australia \\ ${ }^{8}$ Australian Prostate Cancer Research Centre - Queensland, Brisbane, Queensland, Australia \\ ${ }^{9}$ Department of Diabetes and Endocrinology, Princess Alexandra Hospital, Brisbane, Queensland, Australia \\ ${ }^{10}$ School of Medicine, The University of Queensland, Brisbane, Queensland, Australia \\ ${ }^{11}$ Division of Cancer Services, Princess Alexandra Hospital, Brisbane, Queensland, Australia \\ ${ }^{12}$ Institute of Health and Biomedical Innovation, Queensland University of Technology, Brisbane, \\ Queensland, Australia
}

Correspondence

should be addressed

to L Teleni

Email

laisa.teleni@qut.edu.au

\begin{abstract}
Men receiving androgen deprivation therapy (ADT) for prostate cancer ( $\mathrm{PCa}$ ) are likely to develop metabolic conditions such as diabetes, cardiovascular disease, abdominal obesity and osteoporosis. Other treatment-related side effects adversely influence quality of life (QoL) including vasomotor distress, depression, anxiety, mood swings, poor sleep quality and compromised sexual function. The objective of this study was to systematically review the nature and effects of dietary and exercise interventions on QoL, androgen deprivation symptoms and metabolic risk factors in men with PCa undergoing ADT. An electronic search of CINAHL, CENTRAL, Medline, PsychINFO and reference lists was performed to identify peerreviewed articles published between January 2004 and December 2014 in English. Eligible study designs included randomised controlled trials (RCTs) with pre- and post-intervention data. Data extraction and assessment of methodological quality with the Cochrane approach was conducted by two independent reviewers. Seven exercise studies were identified. Exercise significantly improved QoL, but showed no effect on metabolic risk factors (weight, waist circumference, lean or fat mass, blood pressure and lipid profile). Two dietary studies were identified, both of which tested soy supplements. Soy supplementation did not improve any outcomes. No dietary counselling studies were identified. No studies evaluated androgen-deficiency symptoms (libido, erectile function, sleep quality, mood swings, depression, anxiety and bone mineral density). Evidence from RCTs indicates that exercise
\end{abstract}

\footnotetext{
Key Words

- prostate cancer

- androgen deprivation therapy

- quality of life

diet

- aerobic exercise

- resistance exercise
} 
enhances health- and disease-specific QoL in men with PCa undergoing ADT. Further studies are required to evaluate the effect of exercise and dietary interventions on QoL, androgen deprivation symptoms and metabolic risk factors in this cohort.

\section{Introduction}

Prostate cancer (PCa) is the second most commonly diagnosed cancer in men after lung cancers. According to the International Agency for Research on Cancer, more than 1.1 million cases were diagnosed in 2012, with highest incidence rates in Australia, New Zealand and Northern America followed by Western and Northern Europe (Ferlay et al. 2013).

Managing treatment-related morbidity is increasingly important for men undergoing androgen deprivation therapy (ADT). In hormone-sensitive PCa, androgens can stimulate the growth of cancer cells. These effects can be blocked or reduced through the use of surgical or medical ADT, shrinking the tumour or slowing tumour growth. This therapy is common for the management of metastatic PCa and as an adjuvant therapy in localised PCa. With the exception of primary ADT in men with localised PCa (Sammon et al. 2015), the use rates of ADT in Australia and the USA are rising, with this therapy being offered earlier in the cancer trajectory (Grossmann et al. 2011). Compared with no treatment, ADT is associated with an increased risk in non-cancer mortality in men over 66 years with localised disease (Abdollah et al. 2015). These men are more likely to develop metabolic conditions, such as diabetes, cardiovascular disease and abdominal obesity, or experience further life-limiting morbidities such as osteoporosis. Treatment-related side effects include vasomotor distress, depression and anxiety, mood swings, poor sleep quality and compromised libido and erectile function leading to diminished function and quality of life (QoL; Grossmann et al. 2011).

Evidence indicates that the effects of ADT can be attenuated through specific health behaviours. The current 'Nutrition and physical activity guidelines for cancer survivors' (Rock et al. 2012) recommend that for all cancers, nutrition, physical activity, stress and sleep assessment should commence as soon as possible after diagnosis, and account for current and anticipated preferences, symptoms and lifestyle needs. In cancer survivors, exercise has been shown to improve cardiovascular fitness, muscle strength, body composition, fatigue, anxiety, depression and some aspects of QoL
(Rock et al. 2012). Similarly, an individualised multidisciplinary approach including dietetics and exercise physiotherapy was recommended in the joint consensus statement from the Endocrine Society of Australia, the Australian and New Zealand Bone and Mineral Society, and the Urological Society of Australia and New Zealand (Grossmann et al. 2011).

In a previous meta-analysis, Chipperfield et al. (2014) evaluated the effect of physical activity on outcomes such as depression, anxiety, cognitive function and QoL while excluding studies of other outcomes or those including dietary interventions. There are no meta-analyses of high level evidence that evaluate the effect of dietary and/or exercise interventions in preventing or ameliorating ADTrelated metabolic and other side effects beyond those relating to QoL.

The aim of this review is to examine the effectiveness of dietary and/or exercise in preventing, modifying or managing the effects of ADT in men with PCa. Specifically, the primary objective is to investigate the effect of these interventions on QoL, and the secondary objectives are to evaluate the effects on androgen deprivation symptoms and metabolic risk factors.

\section{Materials and methods}

\section{Eligibility criteria}

To address the review objectives, we included randomised controlled trials (RCTs) of adult male participants $(>18$ years of age) with PCa undergoing ADT. Studies with participants not undergoing ADT, and whose data were reported separately, were included. Interventions of interest compared aerobic exercise, resistance exercise and/or dietary counselling with standard care or no treatment. Dietary supplements were compared with matched placebo. Primary outcome measures were health-related QoL (overall physical or mental health) and disease-specific QoL (QoL in the context of PCa) as measured by validated questionnaires. Secondary outcome measures of androgen deprivation symptoms were dual X-ray absorptiometry (DXA) scans of bone mineral

Published by Bioscientifica Ltd. 
density, vasomotor symptoms, insomnia and mood swings, weight gain, depression and anxiety. Secondary outcome measures of metabolic risk factors were fasting glucose, fasting lipid profile and/or fat mass measured by DXA.

\section{Search methods}

A search of Medline was undertaken (Table 1) to identify relevant text words and index terms used to describe the articles. A second search of CINAHL, Cochrane CENTRAL, Medline and PsychINFO was conducted using identified text words and index terms. Reference lists of relevant articles were hand searched for additional studies that met the inclusion criteria. Only articles published in the English language between 2004 and 2014 were included.

\section{Data collection and extraction}

One author (L Teleni) analysed the titles and abstracts from the searches. Full text was sought for studies that both potentially met the eligibility criteria and, where eligibility could not be determined due to insufficient information. Two authors (A L McCarthy and L Teleni) independently assessed eligibility from the full text; any disagreements were resolved by discussion and consensus.

Two review authors (A L McCarthy and L Teleni) independently extracted data from each study using a template developed by the authors for the purpose of the review. Data extracted included descriptions of general study information, methods, participants, intervention and comparator, outcomes and length of follow-up, study results for each outcome and time of assessment. Any discrepancies between review authors were discussed and corrected by consulting the original article. For studies published more than once, data was collated. Where data for an outcome was reported more than once, the data from the publication with the largest study population was used.

\section{Assessment of risk or bias}

Two review authors (A L McCarthy and L Teleni) independently assessed the risk of bias of the included studies using the Cochrane Collaboration's tool for assessing risk of bias (Higgins \& Green 2011). This tool assesses random sequence generation, allocation concealment, blinding of participants and personnel, blinding of outcome assessment, incomplete outcome data and selective reporting. Each study was graded according to low, high or unclear risk of bias.

\section{Statistical analysis}

Where possible, data were used to calculate the mean difference (MD) using the mean for continuous outcomes with number of participants as the denominator for each outcome. Where different scales were used, the standardised MD (SMD) was calculated. Where the s.D. of the change scores was not given, they were imputed using the S.D. from similar studies (Higgins \& Green 2011).

Obvious clinical heterogeneity was assessed by comparing populations, settings, interventions and outcomes before deciding whether it was appropriate to pool studies. Where pooling was undertaken, statistical heterogeneity was assessed with the $I^{2}$ statistic. Where it was reasonable to assume a single pooled effect $\left(I^{2}<50 \%\right)$, a fixed-effect model was used. Where variation in populations and interventions or substantial heterogeneity $\left(I^{2} \geq 50 \%\right)$ was evident, a random-effects model was used (DerSimonian \& Laird 1986). Review Manager 5.3 was used for statistical analysis (RevMan version 5.3, The

Table 1 Mesh and keywords used to search for publications in Ovid MEDLINE from 1946 to present

Subject
Prostate cancer
Dietary interventions
Fietary supplements
Dairy, meat and fat
Exercise interventions
Search limits

\section{MeSH and keywords}

'Prostatic Neoplasms' (MeSH) or prostate cancer 'Diet' (MeSH) or 'Diet Therapy' (MeSH)

'Isoflavones' (MeSH) or isoflavones or 'Flax' (MeSH) or flaxseed or 'Soy Milk' (MeSH) or 'Soy Foods' (MeSH)/or soy or 'Soybean Proteins' (MeSH) or 'Carotenoids' (MeSH) or 'Vitamin E' (MeSH) or lycopene or 'Folic Acid' (MeSH) or folate or 'Dietary Supplements' (MeSH) or 'Complementary Therapies' (MeSH) or 'Naturopathy' (MeSH)

'Fruit' (MeSH) or fruit or 'Vegetables' (MeSH) or vegetables or 'Dietary Fiber' (MeSH) or dietary fibre 'Dairy Products' (MeSH) or dairy or 'Calcium, Dietary' (MeSH) or 'Meat' (MeSH) or 'Dietary Fats' (MeSH)

'Exercise' (MeSH) or 'physical endurance' (MeSH) or 'Exercise Therapy' (MeSH)

English language, 2004-current, all adult (19+ years) 
Nordic Cochrane Centre, The Cochrane Collaboration, Copenhagen, Denmark). Where statistical pooling was not possible, findings are presented in narrative form.

\section{Results}

Of the 246 potential articles, 13 met the inclusion criteria (Fig. 1), providing data for nine intervention studies. Intervention characteristics and key findings are summarised in Table 2. The median sample size was 100 (33-121) participants with median intervention duration of 12 (12-24) weeks. Although participant eligibility criteria were similar, cancer stage and treatment varied between studies, as did the depth of information provided about these variables. Only three of the nine studies provided details on the duration of ADT (Fig. 2). As expected for lifestyle-based interventions, the largest risk of bias was lack of blinding of participants.

There were also studies that lacked adequate information for domains of risk of bias assessment. Lack of details pertaining to both random sequence generation and allocation of concealment potentially introduced selection bias. Where studies failed to report blinding of outcome assessors, they introduced potential detection bias. In addition, incomplete outcome data and selective reporting may have increased the risk of attrition and reporting bias.

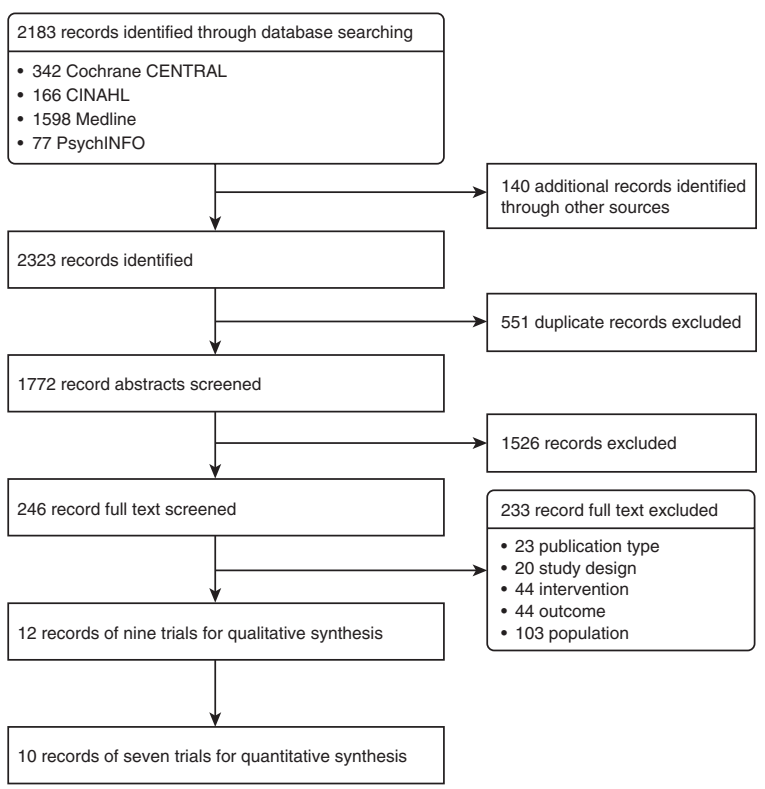

Figure 1

PRISMA flowchart.

\section{Interventions}

Seven RCTs investigated the effects of exercise on QoL, androgen deprivation symptoms and/or metabolic risk factors in men with PCa (Segal et al. 2009, Culos-Reed et al. 2010, Galvao et al. 2010, 2014, Bourke et al. 2011, 2014, Alberga et al. 2012, Cormie et al. 2013, Uth et al. 2014). Four studies included intervention groups that combined resistance exercise training (RET) and aerobic exercise training (AET) (Galvao et al. 2010, 2014, Bourke et al. 2011, 2014), two studies used only RET (Segal et al. 2009, Culos-Reed et al. 2010, Alberga et al. 2012), one study used AET (Segal et al. 2009, Alberga et al. 2012) and one used football training sessions (Uth et al. 2014). Participants in the interventions involving AET, trained at an intensity ranging from 55 to $85 \%$ of maximal heart rate or 11-15 points on the Borg Rating of Perceived Exertion Scale. Most studies of RET did not report exercise intensity. Of those that did, participants trained at $60-70 \%$ of one repetition maximum.

Two dietary RCTs of 99 participants investigated the efficacy of 12 weeks of soy supplementation on QoL, androgen deprivation symptoms or metabolic risk factors in men with PCa (Sharma et al. 2009, Napora et al. 2011, Vitolins et al. 2013). One study had four treatment groups randomising venlafaxine and venlafaxine placebo as well as soy and soy placebo (Vitolins et al. 2013). For this review, we only included the treatment groups where venlafaxine placebos were used so as to compare the effects of soy supplementation.

\section{Quality of life}

Health-related QoL was reported in five exercise studies of 427 participants (Culos-Reed et al. 2010, Galvao et al. 2010, 2014, Bourke et al. 2011, Alberga et al. 2012). Diseasespecific QoL was reported in three exercise studies of 271 participants (Bourke et al. 2011, 2014, Alberga et al. 2012). There was no significant clinical or statistical heterogeneity $\left(I^{2}=0 \%\right)$ and the overall risk of bias was low. Quantitative analysis showed that exercise improved health-related QoL $(\mathrm{SMD}=0.29 ; 95 \% \mathrm{CI}=0.10-0.49)$ and disease-specific QoL (SMD $=0.36$; 95\% CI $=0.11-0.61)$ in men with PCa undergoing ADT (Figs 3 and 4 respectively).

Health-related QoL was reported in both dietary intervention studies. There was no significant clinical or statistical heterogeneity $\left(I^{2}=0 \%\right)$ and the risk of bias was low. Soy supplementation did not significantly improve health-related QoL $(\mathrm{SMD}=0.01 ; 95 \% \mathrm{CI}=-0.38$ to 0.41$)$. Only Vitolins et al. (2013) evaluated disease-specific QoL,

Published by Bioscientifica Ltd. 

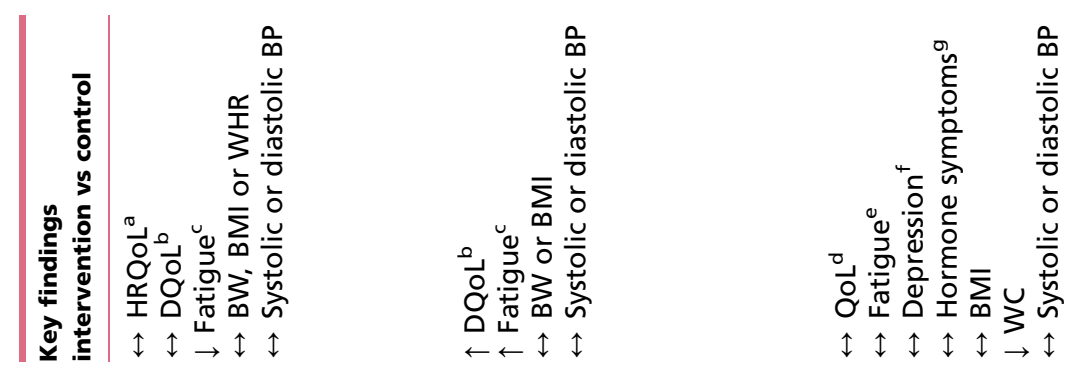

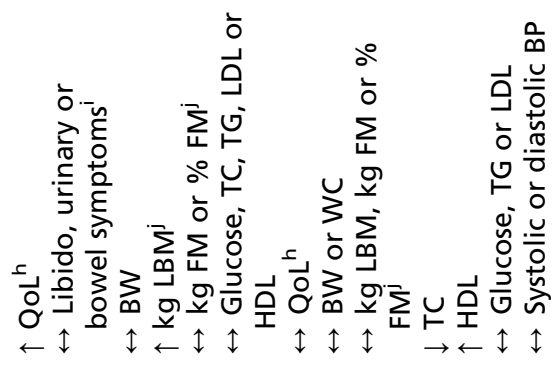
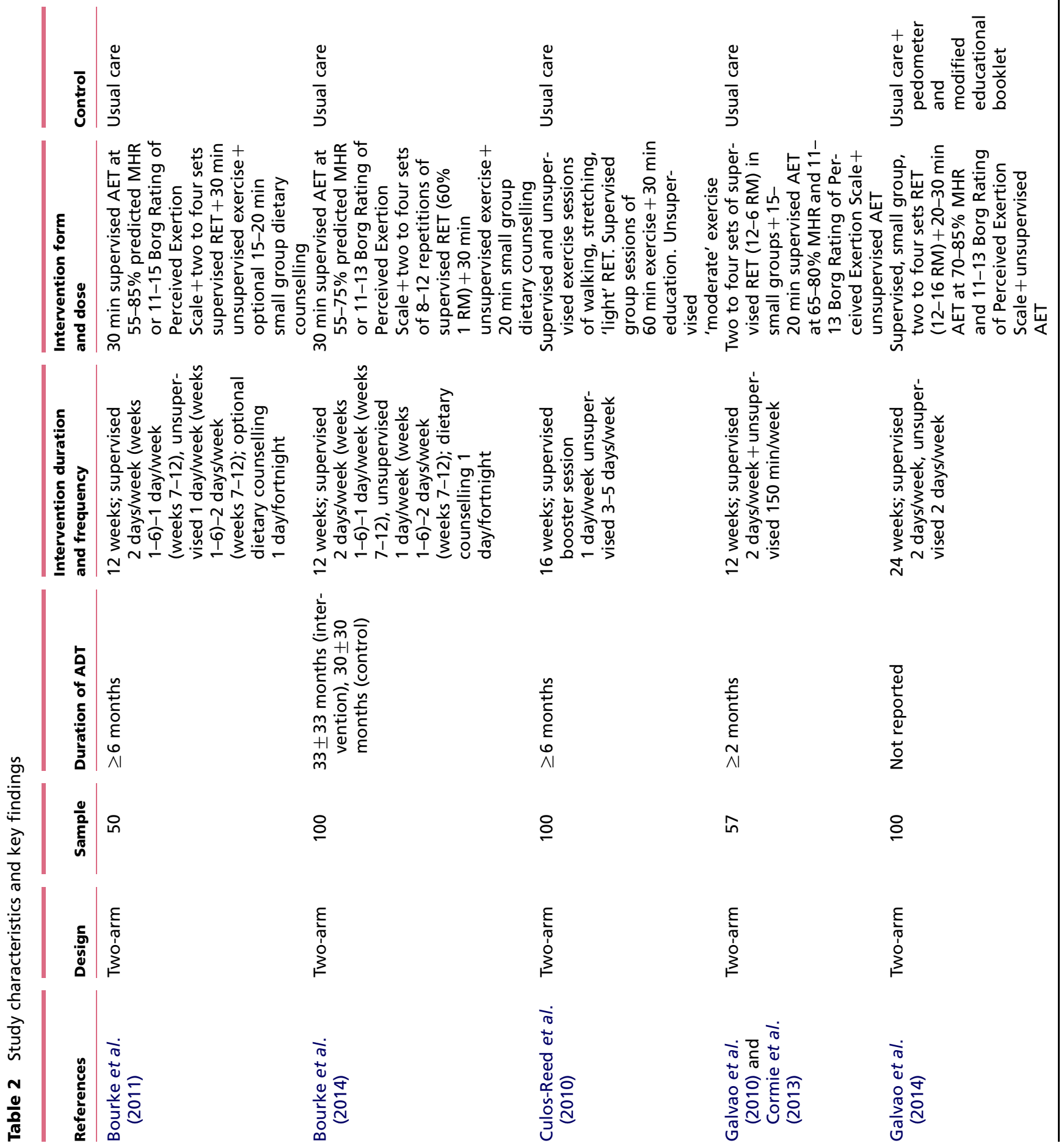

$\stackrel{\circ}{\circ}$

$\xi$
$⿱ 亠$
$\vdots$
$\vdots$
$\vdots$
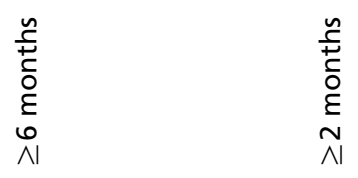

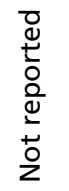

웅

in

음

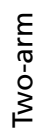

$\varepsilon$
$\frac{1}{5}$
$\vdots$
$\vdots$

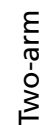




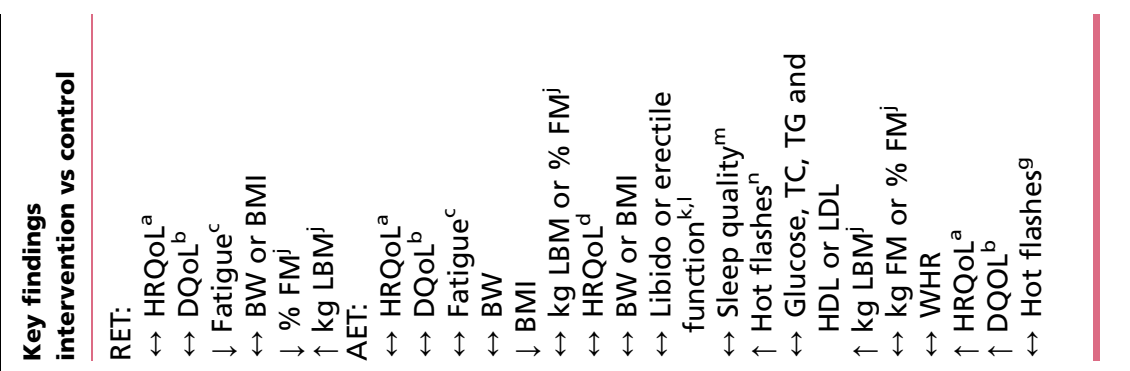

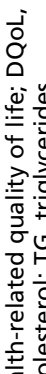

ปั

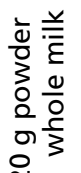

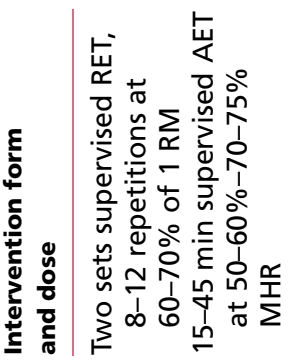

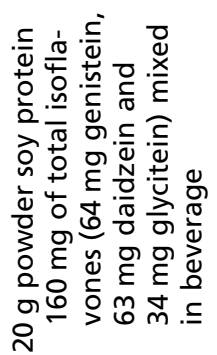

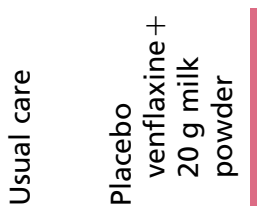
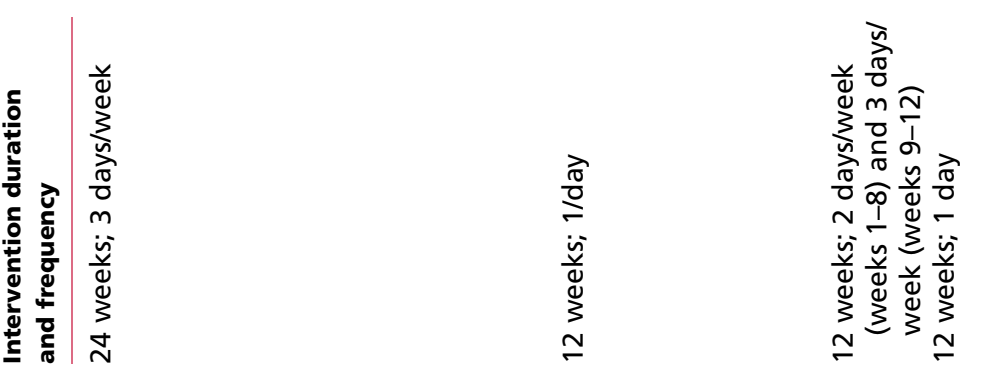

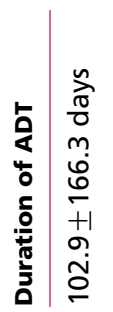

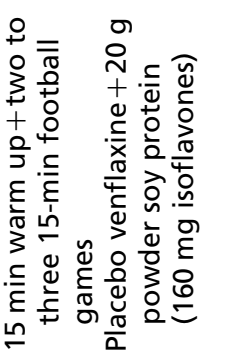

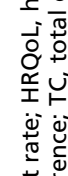

๓ั

m

in 오

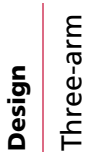

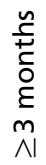

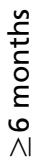

ำ

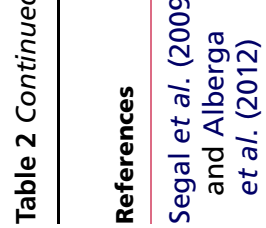

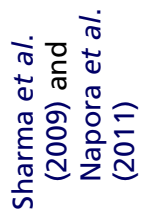

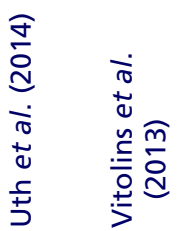

䍃

है

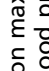

들

d

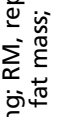

कiष

施

焉

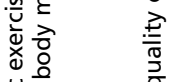

능 융

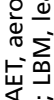

告

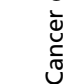

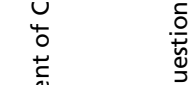

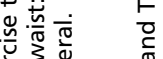

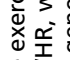

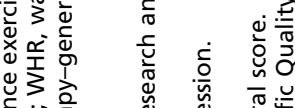

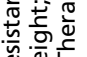

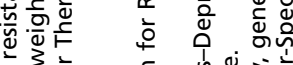

岁

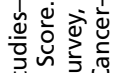

$\xi$
$\substack{\sigma \\ \vdots \\ j}$

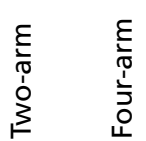

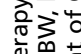

出初

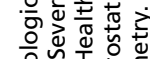

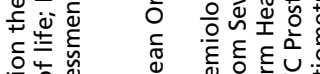

엉

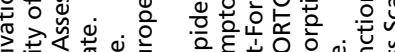

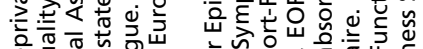

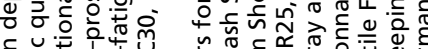

ब. 


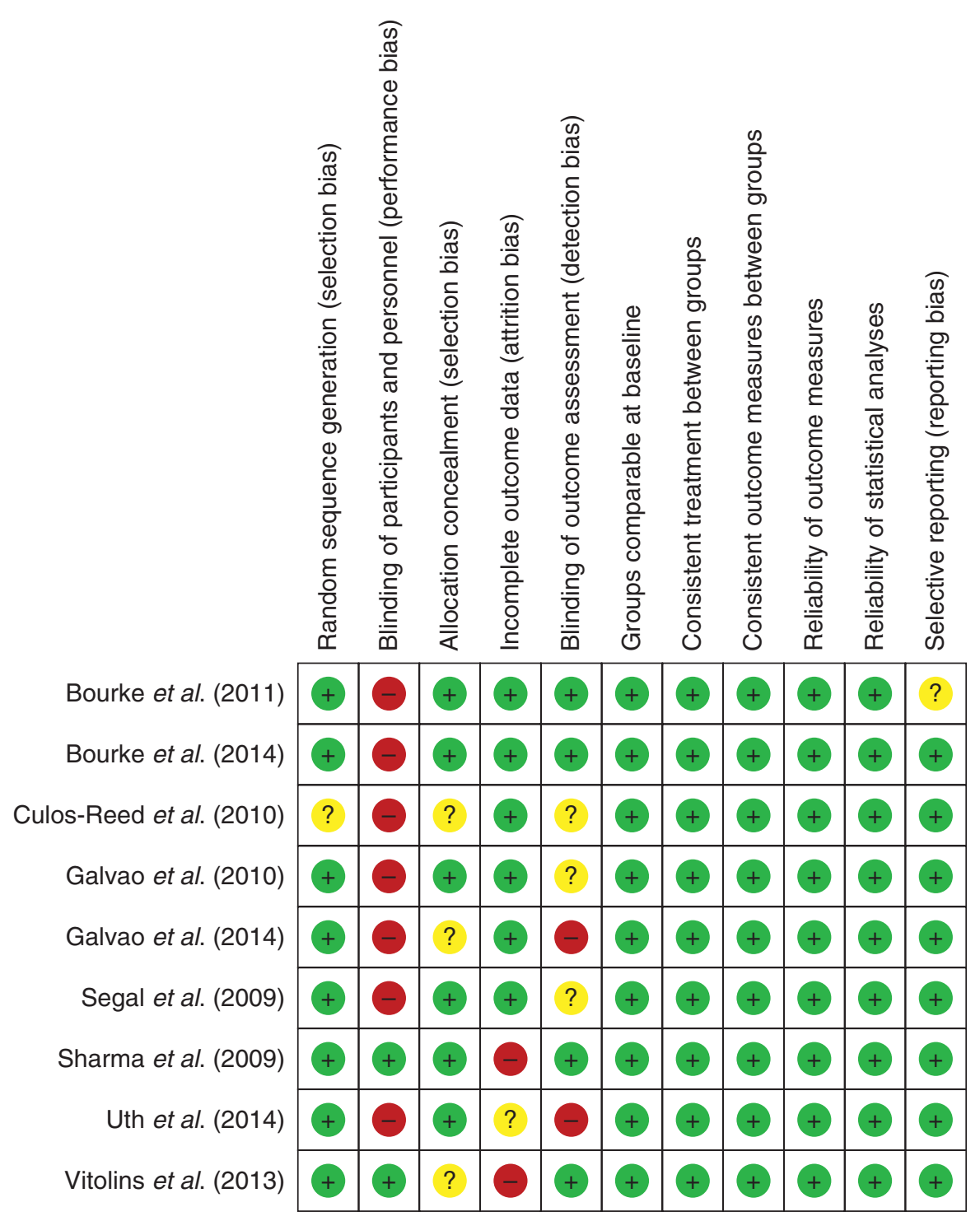

Figure 2

Risk of bias summary. Red (-), high risk of bias; yellow (?), unclear risk of bias; green (+) low risk of bias.

reporting that soy supplementation significantly improved FACT-P global score vs placebo $(112.5 \pm 6.0$ vs $103.8 \pm 6.2, P=0.048)$.

\section{Androgen deprivation symptoms}

No exercise intervention evaluated libido, erectile function, sleep quality and insomnia, mood swings, depression or anxiety or bone mineral density. Both dietary intervention studies evaluated the effect of soy supplementation on vasomotor distress. Sharma et al. (2009) reported a significant difference in hot flash scores between groups, but within-group analysis showed no significant improvement in vasomotor distress score in either the placebo or the soy arms due to the imbalance in scores at baseline. Similarly, Vitolins et al. (2013) found soy supplementation did not significantly improve hot flash number, severity or hot flash score vs placebo. One dietary intervention study evaluated libido, erectile function and sleep quality but found no significant improvements with soy supplementation (Sharma et al. 2009, Napora et al. 2011). No dietary studies evaluated insomnia, mood swings, depression, anxiety or bone mineral density. 


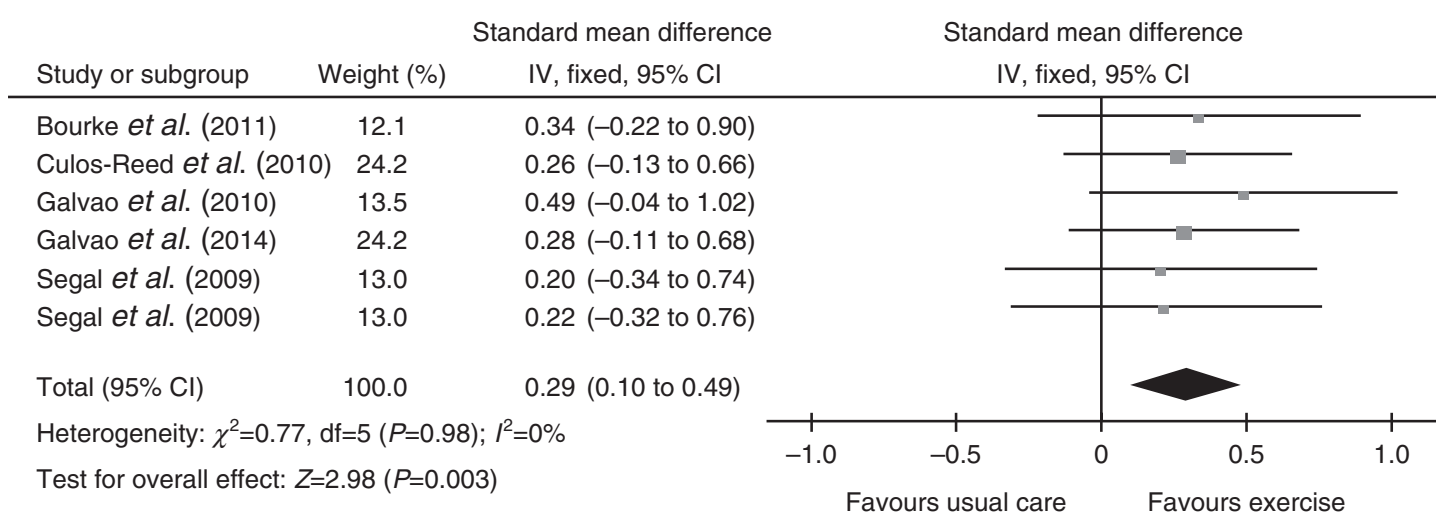

Figure 3

Forest plot of comparison: exercise vs usual care on health-related quality of life.

\section{Metabolic risk factors}

Four exercise intervention studies totalling 310 participants evaluated the effect of exercise on body weight. There was no significant clinical or statistical heterogeneity $\left(I^{2}=0 \%\right)$ and the overall risk of bias was low. Quantitative analysis showed that exercise did not significantly improve total body weight $(\mathrm{MD}=0.26 ; 95 \%$ $\mathrm{CI}=-2.40$ to 2.93; Galvao et al. 2010, 2014, Bourke et al. 2011, 2014). One exercise intervention study evaluated waist:hip ratio, but showed no differences between the intervention and control groups (Bourke et al. 2011). Similarly, exercise did not significantly improve waist circumference measures, which were reported in two studies with a combined total of 200 participants $(\mathrm{MD}=-0.38 ; 95 \% \mathrm{CI}=2.97$ to 2.22; Culos-Reed et al. 2010, Galvao et al. 2014). Body composition, as measured by DXA, was reported in seven exercise intervention studies. There was no significant clinical or statistical heterogeneity $\left(I^{2}=0 \%\right)$ and the overall risk of bias was low. Exercise did not significantly improve any body composition measure including lean body mass $(\mathrm{MD}=-0.20 ; 95 \% \mathrm{CI}=-1.72$ to 1.32 ; Galvao et al. 2010, 2014, Alberga et al. 2012, Uth et al. 2014), total fat mass $(\mathrm{MD}=-0.61 ; 95 \% \mathrm{CI}=-2.48$ to 1.26 ; Galvao et al. 2010, 2014, Uth et al. 2014) or percentage fat mass $(\mathrm{MD}=-0.71 ; 95 \% \mathrm{CI}=-1.96$ to 0.55 ; Galvao et al. 2010, 2014, Alberga et al. 2012, Uth et al. 2014).

One dietary intervention study evaluated body weight and BMI but found no significant improvements with soy supplementation (Sharma et al. 2009, Napora et al. 2011). Neither dietary intervention evaluated the effect of soy supplementation on body composition.

Three intervention studies with a combined total of 300 participants reported that exercise did not significantly improve systolic blood pressure $(\mathrm{MD}=1.72 ; 95 \%$ $\mathrm{CI}=-2.47$ to 5.90; Culos-Reed et al. 2010, Galvao et al. 2010, Bourke et al. 2014). Similarly, exercise did not significantly improve blood glucose levels $(\mathrm{MD}=0.13$; $95 \% \mathrm{CI}=-0.16$ to 0.43$)$, total cholesterol $(\mathrm{MD}=0.13$; $95 \% \mathrm{CI}=-0.18$ to 0.44$)$, triglycerides $(\mathrm{MD}=-0.06$;

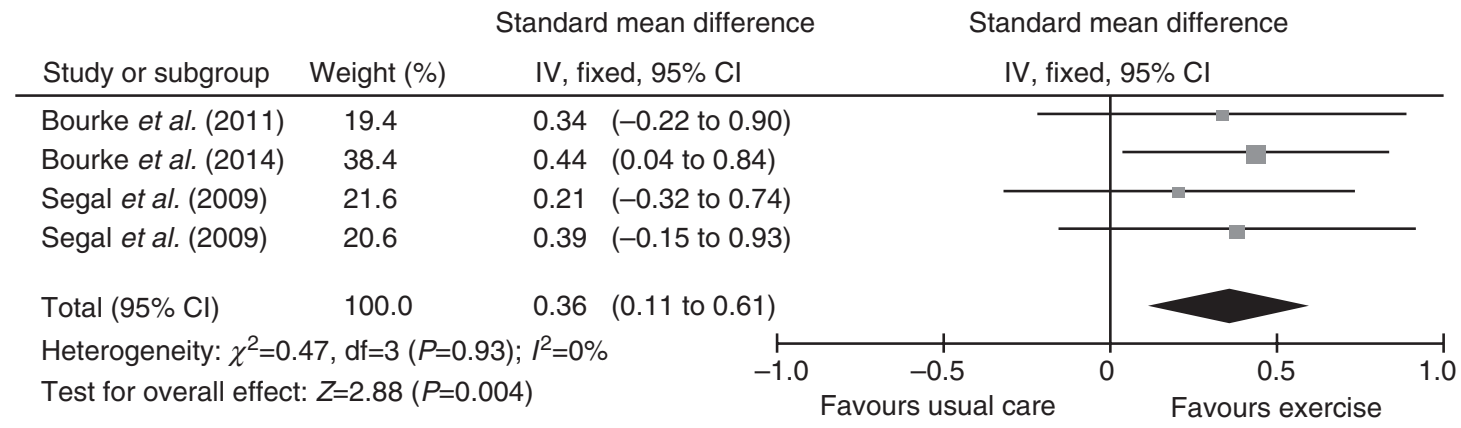

Figure 4

Forest plot of comparison: exercise vs usual care on disease-specific quality of life. 
$95 \% \mathrm{CI}=-0.27$ to 0.15$)$, $\mathrm{LDL}$ cholesterol $(\mathrm{MD}=0.06$; $95 \% \mathrm{CI}=-0.20$ to 0.32$)$ or HDL cholesterol $(\mathrm{MD}=0.06$; $95 \% \mathrm{CI}=-0.05$ to 0.16$)$.

Only one dietary intervention study evaluated the effect of soy supplementation on lipid profile. Soy supplementation did not significantly improve total cholesterol, triglycerides, LDL cholesterol or HDL cholesterol. Neither dietary supplement studies evaluated the effect of soy on glucose levels.

\section{Discussion}

\section{Exercise}

Exercise interventions conducted over 12-24 weeks and which consisted of two to three days per week of combined aerobic and resistance exercise, were associated with significant improvements in health- and disease-specific QoL in men with PCa receiving ADT. Despite these improvements, the magnitude of effect of exercise on these outcomes was small to moderate. The majority of included studies implemented resistance and aerobic exercise as a combined intervention, so it was not possible to determine whether this improvement was attributable to the one type of exercise or the synergy of both. These results are consistent with the meta-analysis by Chipperfield et al. (2014) who reported preliminary data indicating that physical activity significantly improved QoL. However, in the present analysis there were no corresponding improvements in any secondary outcomes. A possible explanation lies in the studies' designs. Exercise intervention studies are often at much greater risk of performance bias compared with other randomised trial designs due to the use of usual care or waitlist control groups, as well as the logistical inability to blind participants and interventionists. Moreover, those in the exercise groups had longer and more intense contact with interventionists, a degree of health professional contact that could have improved perceptions of QoL independent of the effect of exercise. Therefore, improvements in these outcomes may have been masked by a variability in the types of exercise, intensity of the intervention and the duration of the interventions.

Bone fracture is a major risk factor for increased mortality. Depleted testosterone and oestrogen levels in men with PCa undergoing ADT can lead to bone loss (Higano 2004). These changes increase bone resorption and suppress bone formation, increasing the risk of fracture (Higano 2008, Michaud 2010). Despite the wellestablished effect of ADT on bone mineral density, there were a notable lack of studies evaluating the effect of exercise on this outcome. Similarly, other androgen deficiency symptoms including hot flashes, sexual function, sleep quality, depression and anxiety were often not reported as outcomes. These findings are consistent with Chipperfield et al. (2014) who also identified no studies evaluating the effect of physical activity on anxiety and depression.

Although ADT is associated with reduced PCa-specific mortality, its association with increased risk of non-cancer mortality, metabolic parameters and body composition could lead to the development of metabolic syndrome. Metabolic syndrome is a cluster of cardiovascular risk factors including insulin resistance, increased triglycerides and fasting glucose, low HDL, increased waist circumference and hypertension. It is implicated in the development of diabetes and cardiovascular disease. In addition to metabolic syndrome, large cohort analyses have demonstrated that serum cholesterol is independently associated with cardiovascular mortality (Stamler et al. 1986, Lewington et al. 2007).

Interestingly, although associated with the development of diabetes (Saylor \& Smith 2009), the effect of ADT on the risk of developing cardiovascular disease is unclear. ADT increases a number of cardiovascular risk factors, including elevated fasting insulin; decreased insulin sensitivity; worsened lipid profile (Whitsel et al. 2001, Isidori et al. 2005, Laughlin et al. 2008, Traish et al. 2009); hypertension (Svartberg et al. 2004) and abdominal obesity (Marin et al. 1993). In a large meta-analysis of observational studies, Zhao et al. (2014) reported a nonsignificant $10 \%$ increase in cardiovascular disease risk (hazard ratio $(\mathrm{HR})=1.10 ; 95 \% \mathrm{CI}=1.00-1.21 ; P=0.06$ ) but a significant association with cardiovascular mortality $(\mathrm{HR}=1.17 ; 95 \% \mathrm{CI}=1.04-1.32 ; P=0.01)$. Conversely, in a meta-analysis of RCTs (Nguyen et al. 2011), the relative risk of cardiovascular death for men undergoing ADT for PCa vs control was not significant (risk ratio $(\mathrm{RR})=0.93$; 95\% CI $=0.79-1.10 ; P=0.41)$. The discrepancy between these two analyses could be explained by differences in risk. Compared with patients with more advanced disease, low risk patients have a higher life expectancy and are less likely to die of their cancer and therefore live long enough for cardiovascular disease to become a problem.

We found that exercise did not significantly improve lipid profiles, blood glucose levels, blood pressure, body weight or body composition. It is possible that too few studies were included in the current meta-analysis, thereby providing insufficient data or sample size to

Published by Bioscientifica Ltd. 
identify any ameliorative effect of exercise on these outcomes.

Weight gain in men undergoing ADT for PCa over 12-24 weeks would likely be minimal. Seible et al. (2014) reported that even one year after ADT initiation, weight gain was clinically insignificant $(1.32 \pm 4 \mathrm{~kg}, P=0.0005)$. Contrary to traditional beliefs about risk groups, Seible et al. (2014) has previously reported that the independent predictors of weight gain in this population include a nonobese BMI and a relatively young age (i.e. $<30 \mathrm{~kg} / \mathrm{m}^{2}$ and $<65$ years old respectively). The mean sample ages of the studies analysed in this review were over 65 years, with most centring around 70 years. The inclusion of studies of older populations could have unintentionally obfuscated any larger weight alterations in the younger participants, resulting in smaller mean weight changes.

It is also possible that any losses in fat mass may have been obscured by gains in lean tissue. In men undergoing ADT, significant weight and body composition changes tend to occur within the first few months of treatment initiation and continue for 1-2 years. In an observational study, Smith et al. (2002) reported that men undergoing 48 weeks of ADT experienced significant increases in percentage body fat $(9.4 \pm 1.7 \%, P<0.001)$ and significant decreases in percentage lean body mass $(2.7 \pm 0.5 \%$, $P<0.001)$. In the current study, despite most studies' eligibility criteria including men who had been undergoing ADT for at least 2-6 months, exercise did not significantly improve fat mass or lean body mass. However, similar to weight changes, it is possible that too few studies were included in the current analysis to capture such small changes or that intervention periods were too short to capture the effect of exercise on body composition.

\section{Diet}

The precise contribution of sex steroid deprivation to the adverse effects of ADT is unclear. Low testosterone has been implicated in diminished QoL, androgen-deficiency symptoms and metabolic risk factors in men with PCa on ADT; however, these men also have low oestradiol levels (Sharma et al. 2009). It is hypothesised that due to the structural similarity of isoflavones (soy protein) to oestrogen, soy could induce a weak oestrogenic effect, minimising these adverse effects. In this review, soy supplementation did not significantly improve healthrelated QoL and there were insufficient data to quantitatively address disease-specific QoL. There were also insufficient data to evaluate the effect of soy on androgen deficiency symptoms, with the exception of vasomotor distress. Consistent with previous trials, soy supplementation did not significantly improve vasomotor distress. In breast cancer, soy has been shown to have no effect on the menopausal symptoms of postmenopausal women (Quella et al. 2000, Van Patten et al. 2002, MacGregor et al. 2005). There were insufficient data to evaluate the effect of soy on metabolic risk factors.

\section{Strengths and limitations}

This systematic review includes a quantitative analysis of RCTs, the highest level of evidence for interventions. Where studies were combined, there was minimal clinical and statistical heterogeneity. Data extraction and risk of bias assessment was conducted independently by two reviewers and almost all studies were found to have a low risk of bias.

The findings of this review were limited, however, by the lack of dietary intervention studies, particularly those using dietary counselling. Exercise interventions could not be evaluated by exercise type (i.e. resistance vs aerobic) so any significant results should be considered preliminary and attributed to combined modality.

\section{Conclusion}

There are significant gaps in the literature with regards to dietary and exercise interventions for the management of QoL, androgen deficiency symptoms and metabolic risk factors in men receiving ADT for PCa. Although the effects of exercise interventions on QoL are apparent, it is unclear whether these improvements are attributable to increased interventionist contact, aerobic exercise, resistance exercise or the combination of all three. More studies are required that control for interventionist contact as well as exercise type (i.e. aerobic or resistance), intensity and duration.

There is also a clear gap in the literature as to the effect of dietary counselling on QoL, androgen deficiency symptoms and metabolic risk factors in men undergoing ADT. No dietary studies addressing the effects of dietary counselling on ADT symptom management were identified for this review. It is possible that either the dietary counselling studies are addressing outcomes which have little relevance to ADT symptom management or they do not exist.

Despite the findings of this review, interventions that are effective in this population are still needed to ameliorate or manage the adverse effects of ADT. This is

Published by Bioscientifica Ltd. 
particularly important for decreasing this risk of cardiovascular disease and diabetes through managing metabolic risk factors and addressing bone mineral density.

\section{Declaration of interest}

The authors declare that there is no conflict of interest that could be perceived as prejudicing the impartiality of the research reported.

\section{Funding}

This work was supported by the New Professor Grant, Queensland University of Technology.

\section{Author contribution statement}

A L McCarthy conceptualised the systematic review. A L McCarthy and $L$ Teleni designed the systematic review and collected the data. L Teleni, A L McCarthy and R J Chan performed the initial analysis and interpretation and wrote the manuscript. All authors reviewed the analysis, interpretation and critically reviewed the manuscript. All authors have read and approved the version of the manuscript being submitted.

\section{References}

Abdollah F, Sammon JD, Reznor G, Sood A, Schmid M, Klett DE, Sun M, Aizer AA, Choueiri TK, Hu JC et al. 2015 Medical androgen deprivation therapy and increased non-cancer mortality in non-metastatic prostate cancer patients aged $\geq 66$ years. European Journal of Surgical Oncology $\mathbf{4 1}$ 1529-1539. (doi:10.1016/j.ejso.2015.06.011)

Alberga AS, Segal RJ, Reid RD, Scott CG, Sigal RJ, Khandwala F, Jaffey J, Wells GA \& Kenny GP 2012 Age and androgen-deprivation therapy on exercise outcomes in men with prostate cancer. Supportive Care in Cancer 20 971-981. (doi:10.1007/s00520-011-1169-x)

Bourke L, Doll H, Crank H, Daley A, Rosario D \& Saxton JM 2011 Lifestyle intervention in men with advanced prostate cancer receiving androgen suppression therapy: a feasibility study. Cancer Epidemiology, Biomarkers \& Prevention 20 647-657. (doi:10.1158/1055-9965.EPI-10-1143)

Bourke L, Gilbert S, Hooper R, Steed LA, Joshi M, Catto JWF, Saxton JM \& Rosario DJ 2014 Lifestyle changes for improving disease-specific quality of life in sedentary men on long-term androgen-deprivation therapy for advanced prostate cancer: a randomised controlled trial. European Urology 65 865-872. (doi:10.1016/j.eururo.2013.09.040)

Chipperfield K, Brooker J, Fletcher J \& Burney S 2014 The impact of physical activity on psychosocial outcomes in men receiving androgen deprivation therapy for prostate cancer: a systematic review. Health Psychology 33 1288-1297. (doi:10.1037/hea0000006)

Cormie P, Newton RU, Taaffe DR, Spry N, Joseph D, Akhlil Hamid M \& Galvao DA 2013 Exercise maintains sexual activity in men undergoing androgen suppression for prostate cancer: a randomized controlled trial. Prostate Cancer and Prostatic Diseases 16 170-175. (doi:10.1038/ pcan.2013.22)

Culos-Reed SN, Robinson JW, Lau H, Stephenson L, Keats M, Norris S, Kline G \& Faris P 2010 Physical activity for men receiving androgen deprivation therapy for prostate cancer: benefits from a 16-week intervention. Supportive Care in Cancer 18 591-599. (doi:10.1007/ s00520-009-0694-3)

DerSimonian R \& Laird N 1986 Meta-analysis in clinical trials. Controlled Clinical Trials 7 177-188. (doi:10.1016/0197-2456(86)90046-2)
Ferlay J, Soerjomataram I, Ervik M, Dikshit R, Eser S, Mathers C, Rebelo M, Parkin DM, Forman D \& Bray F 2013 GLOBOCAN 2012 v1.0, Cancer Incidence and Mortality Worldwide: IARC CancerBase No. 11. Lyon, France: IARC. (available at: http://globocan.iarc.fr)

Galvao DA, Taaffe DR, Spry N, Joseph D \& Newton RU 2010 Combined resistance and aerobic exercise program reverses muscle loss in men undergoing androgen suppression therapy for prostate cancer without bone metastases: a randomized controlled trial. Journal of Clinical Oncology 28 340-347. (doi:10.1200/JCO.2009.23.2488)

Galvao DA, Spry N, Denham J, Taaffe DR, Cormie P, Joseph D, Lamb DS, Chambers SK \& Newton RU 2014 A multicentre year-long randomised controlled trial of exercise training targeting physical functioning in men with prostate cancer previously treated with androgen suppression and radiation from TROG 03.04 radar. European Urology 65 856-864. (doi:10.1016/j.eururo.2013.09.041)

Grossmann M, Hamilton EJ, Gilfillan C, Bolton D, Joon DL \& Zajac JD 2011 Bone and metabolic health in patients with non-metastatic prostate cancer who are receiving androgen deprivation therapy. Medical Journal of Australia 194 301-306.

Higano CS 2004 Understanding treatments for bone loss and bone metastases in patients with prostate cancer: a practical review and guide for the clinician. Urologic Clinics of North America 31 331-352. (doi:10.1016/j.ucl.2004.01.001)

Higano CS 2008 Androgen-deprivation-therapy-induced fractures in men with nonmetastatic prostate cancer: what do we really know? Nature Clinical Practice. Urology 5 24-34. (doi:10.1038/ncpuro0995)

Higgins JPT, Altman DG, Gøtzsche PC, Jüni P, Moher D, Oxman AD, Savović J, Schulz KF, Weeks L \& Sterne JAC 2011 The Cochrane Collaboration's tool for assessing risk of bias in randomised trials. British Medical Journal 343 d5928. (doi:10.1136/bmj.d5928)

Isidori AM, Giannetta E, Greco EA, Gianfrilli D, Bonifacio V, Isidori A, Lenzi A \& Fabbri A 2005 Effects of testosterone on body composition, bone metabolism and serum lipid profile in middle-aged men: a metaanalysis. Clinical Endocrinology 63 280-293. (doi:10.1111/j.1365-2265. 2005.02339.x)

Laughlin GA, Barrett-Connor E \& Bergstrom J 2008 Low serum testosterone and mortality in older men. Journal of Clinical Endocrinology and Metabolism 93 68-75. (doi:10.1210/jc.2007-1792)

Lewington S, Whitlock G, Clarke R, Sherliker P, Emberson J, Halsey J, Qizilbash N, Peto R \& Collins R 2007 Blood cholesterol and vascular mortality by age, sex, and blood pressure: a meta-analysis of individual data from 61 prospective studies with 55,000 vascular deaths. Lancet 370 1829-1839. (doi:10.1016/S0140-6736(07)61778-4)

MacGregor CA, Canney PA, Patterson G, McDonald R \& Paul J 2005 A randomised double-blind controlled trial of oral soy supplements versus placebo for treatment of menopausal symptoms in patients with early breast cancer. European Journal of Cancer $\mathbf{4 1}$ 708-714. (doi:10.1016/j.ejca.2005.01.005)

Marin P, Holmang S, Gustafsson C, Jonsson L, Kvist H, Elander A, Eldh J, Sjostrom L, Holm G \& Bjorntorp P 1993 Androgen treatment of abdominally obese men. Obesity Research $1245-251$. (doi:10.1002/j. 1550-8528.1993.tb00618.x)

Michaud LB 2010 Managing cancer treatment-induced bone loss and osteoporosis in patients with breast or prostate cancer. American Journal of Health-System Pharmacy 67 S20-S30 (quiz S31-S23). (doi:10.2146/ajhp100078)

Napora JK, Short RG, Muller DC, Carlson OD, Odetunde JO, Xu X Carducci M, Travison TG, Maggio M, Egan JM et al. 2011 High-dose isoflavones do not improve metabolic and inflammatory parameters in androgen-deprived men with prostate cancer. Journal of Andrology $\mathbf{3 2}$ 40-48. (doi:10.2164/jandrol.110.010983)

Nguyen PL, Je Y, Schutz FA, Hoffman KE, Hu JC, Parekh A, Beckman JA \& Choueiri TK 2011 Association of androgen deprivation therapy with cardiovascular death in patients with prostate cancer: a meta-analysis of randomized trials. Journal of the American Medical Association $\mathbf{3 0 6}$ 2359-2366. (doi:10.1001/jama.2011.1745) 
Quella SK, Loprinzi CL, Barton DL, Knost JA, Sloan JA, LaVasseur BI, Swan D, Krupp KR, Miller KD \& Novotny PJ 2000 Evaluation of soy phytoestrogens for the treatment of hot flashes in breast cancer survivors: A North Central Cancer Treatment Group Trial. Journal of Clinical Oncology 18 1068-1074.

Rock CL, Doyle C, Demark-Wahnefried W, Meyerhardt J, Courneya KS, Schwartz AL, Bandera EV, Hamilton KK, Grant B, McCullough M et al. 2012 Nutrition and physical activity guidelines for cancer survivors. CA: A Cancer Journal for Clinicians 62 242-274. (doi:10.3322/caac. 21142)

Sammon JD, Abdollah F, Reznor G, Pucheril D, Choueiri TK, Hu JC, Kim SP, Schmid M, Sood A, Sun M et al. 2015 Patterns of declining use and the adverse effect of primary androgen deprivation on all-cause mortality in elderly men with prostate cancer. European Urology 68 32-39. (doi:10.1016/j.eururo.2014.10.018)

Saylor PJ \& Smith MR 2009 Metabolic complications of androgen deprivation therapy for prostate cancer. Journal of Urology 181 1998-2006 (discussion 2007-2008). (doi:10.1016/j.juro.2009.01.047)

Segal RJ, Reid RD, Courneya KS, Sigal RJ, Kenny GP, Prud'Homme DG, Malone SC, Wells GA, Scott CG \& Slovinec D'Angelo ME 2009 Randomized controlled trial of resistance or aerobic exercise in men receiving radiation therapy for prostate cancer. Journal of Clinical Oncology 27 344-351. (doi:10.1200/JCO.2007.15.4963)

Seible DM, Gu X, Hyatt AS, Beard CJ, Choueiri TK, Efstathiou JA, Miyamoto DT, Mitin T, Martin NE, Sweeney CJ et al. 2014 Weight gain on androgen deprivation therapy: which patients are at highest risk? Urology 83 1316-1321. (doi:10.1016/j.urology.2014.02.006)

Sharma P, Wisniewski A, Braga-Basaria M, Xu X, Yep M, Denmeade S, Dobs AS, DeWeese T, Carducci M \& Basaria S 2009 Lack of an effect of high dose isoflavones in men with prostate cancer undergoing androgen deprivation therapy. Journal of Urology 182 2265-2272. (doi:10.1016/j.juro.2009.07.030)

Smith MR, Finkelstein JS, McGovern FJ, Zietman AL, Fallon MA, Schoenfeld DA \& Kantoff PW 2002 Changes in body composition during androgen deprivation therapy for prostate cancer. Journal of Clinical Endocrinology and Metabolism 87 599-603. (doi:10.1210/jcem.87.2.8299)
Stamler J, Wentworth D \& Neaton JD 1986 Is relationship between serum cholesterol and risk of premature death from coronary heart disease continuous and graded? Findings in 356222 primary screenees of the Multiple Risk Factor Intervention Trial (MRFIT) Journal of the American Medical Association 256 2823-2828. (doi:10.1001/jama.1986. 03380200061022)

Svartberg J, von Muhlen D, Schirmer H, Barrett-Connor E, Sundfjord J \& Jorde R 2004 Association of endogenous testosterone with blood pressure and left ventricular mass in men. The Tromso Study. European Journal of Endocrinology 150 65-71. (doi:10.1530/eje.0.1500065)

Traish AM, Saad F, Feeley RJ \& Guay A 2009 The dark side of testosterone deficiency: III. Cardiovascular disease. Journal of Andrology 30 477-494. (doi:10.2164/jandrol.108.007245)

Uth J, Hornstrup T, Schmidt JF, Christensen JF, Frandsen C, Christensen KB, Helge EW, Brasso K, Rørth M, Midtgaard J et al. 2014 Football training improves lean body mass in men with prostate cancer undergoing androgen deprivation therapy. Scandinavian Journal of Medicine \& Science in Sports 24 105-112. (doi:10.1111/sms.12260)

Van Patten CL, Olivotto IA, Chambers GK, Gelmon KA, Hislop TG, Templeton E, Wattie A \& Prior JC 2002 Effect of soy phytoestrogens on hot flashes in postmenopausal women with breast cancer: a randomized, controlled clinical trial. Journal of Clinical Oncology 20 1449-1455. (doi:10.1200/JCO.20.6.1449)

Vitolins MZ, Griffin L, Tomlinson WV, Vuky J, Adams PT, Moose D, Frizzell B, Lesser GJ, Naughton M, Radford JE Jr et al. 2013 Randomized trial to assess the impact of venlafaxine and soy protein on hot flashes and quality of life in men with prostate cancer. Journal of Clinical Oncology 31 4092-4098. (doi:10.1200/JCO.2012.48.1432)

Whitsel EA, Boyko EJ, Matsumoto AM, Anawalt BD \& Siscovick DS 2001 Intramuscular testosterone esters and plasma lipids in hypogonadal men: a meta-analysis. American Journal of Medicine 111 261-269. (doi:10.1016/S0002-9343(01)00833-6)

Zhao J, Zhu S, Sun L, Meng F, Zhao L, Zhao Y, Tian H, Li P \& Niu Y 2014 Androgen deprivation therapy for prostate cancer is associated with cardiovascular morbidity and mortality: a meta-analysis of populationbased observational studies. PLOS ONE 9 e107516. (doi:10.1371/ journal.pone.0107516)

Received in final form 16 November 2015

Accepted 19 November 2015

Made available online as an Accepted Preprint

19 November 2015
(C) 2016 Society for Endocrinology Printed in Great Britain
Published by Bioscientifica Ltd. 